\title{
Effect of oxygen on breath markers of oxidative stress
}

\author{
M. Phillips*,\#, R.N. Cataneo*, J. Greenberg*, R. Grodman", , R. Gunawardena", A. Naidu
}

Effect of oxygen on breath markers of oxidative stress. M. Phillips, R.N. Cataneo, J. Greenberg, R. Grodman, R. Gunawardena, A. Naidu. (C) ERS Journals Ltd 2003.

ABSTRACT: Supplemental oxygen is often administered to induce hyperoxia in nonhypoxic patients for indications such as chest pain, despite lack of evidence of clinical benefit. Induced hyperoxia is potentially toxic, since it may increase oxidative stress and peroxidative damage to deoxyribonucleic acid, lipids and proteins.

The aim of this study was to establish whether supplemental oxygen induces oxidative stress in nonhypoxic subjects.

Breath markers of oxidative stress were measured in $\mathbf{3 1}$ healthy subjects before and after breathing $28 \%$ oxygen at $2.0 \mathrm{~L} \cdot \mathrm{min}^{-1}$ via nasal prongs for $30 \mathrm{~min}$ while resting. The criterion standard of oxidative stress was the breath methylated alkane contour (BMAC), a three-dimensional plot of the alveolar gradients of $\mathrm{C4-C20}$ alkanes and monomethylated alkanes produced by lipid peroxidation. Volatile organic compounds (VOCs) in breath were assayed by gas chromatography and mass spectroscopy, and the BMACs before and after oxygenation were compared.

Following oxygenation, there was a significant increase in mean volume under the curve of the BMAC and in alveolar gradients of three VOCs: 3-methyltridecane, 3-methylundecane and 5-methylnonane.

Breath markers of oxidative stress were significantly increased in normal volunteers breathing supplemental oxygen for $30 \mathrm{~min}$.

Eur Respir J 2003; 21: 48-51.

\begin{abstract}
* Menssana Research, Inc., Fort Lee, NJ, "Dept of Medicine, New York Medical College Valhalla, New York, NY, and Dept of Medicine, Saint Vincents Catholic Medical Centers of New York, Staten Island Region, NY, USA.
\end{abstract}

Correspondence: M. Phillips, Menssana Research, Inc., 1 Horizon Road Suite 1415, Fort Lee, NJ 07024, USA. Fax: 12018867004

E-mail: menssana@bellatlantic.net

\section{Keywords: Breath}

oxidative stress

oxygen breathing

volatile organic compounds

Received: June 212002

Accepted after revision: August 142002
Oxygen is a toxic drug. Toxic effects of oxygen were reported in laboratory animals in 1878 but were not recognised in humans until the late 1940s, when an epidemic of blindness in premature newborns was traced to retrolental fibroplasia induced by supplemental oxygen therapy [1]. Oxygen at high partial pressures is now recognised as toxic to the respiratory, cardiovascular, nervous and gastrointestinal systems [2]. The source of oxygen toxicity arises from the mitochondria, where oxygen is the final acceptor of electrons in oxidative metabolism. Oxygen is reduced to water, as well as a number of reactive oxygen species (ROS), including superoxide radical, hydrogen peroxide and hydroxyl radical. These ROS leak out of the mitochondria into the cytoplasm where they cause oxidative damage to a wide variety of biologically important molecules, including deoxyribonucleic acid (DNA), proteins and polyunsaturated fatty acids (PUFAs) in cell membranes [3, 4]. The collective oxidative effect of ROS on biological molecules is termed oxidative stress. It has been implicated as a mechanism of aging [1] and carcinogenesis [5], and is increased in several disorders including rheumatoid arthritis [6], ischaemic heart disease [7] and bronchial asthma [8].

It is a common clinical practice to administer supplemental oxygen in order to induce hyperoxia in nonhypoxic patients with chest pain and suspected acute myocardial infarction. There is little evidence that this is beneficial and it may potentially be harmful by inducing an increase in oxidative stress. However, the effects of oxygen on markers of oxidative stress have not been totally elucidated because previous studies have yielded conflicting results [9-12]. The current authors have recently reported a sensitive new index of oxidative stress, the breath methylated alkane contour (BMAC), comprising a three-dimensional surface plot of the abundance in breath of $\mathrm{C} 4-\mathrm{C} 20$ alkanes and their monomethylated derivatives [13]. In this study, the effects of supplemental oxygen in a group of healthy volunteers, as shown by induced changes in their BMACs, are reported.

\section{Materials and methods}

\section{Human subjects and study design}

Healthy volunteers were recruited from the medical staff of Saint Vincents Catholic Medical Centers of New York (Staten Island Region, NY, USA). The 31 subjects comprised 20 males and 11 females; their mean age was 28.4 yrs (SD 5.0). After an overnight fast, each subject breathed $28 \%$ oxygen at $2.0 \mathrm{~L} \cdot \mathrm{min}^{-1}$ via nasal prongs for $30 \mathrm{~min}$ while resting comfortably. Breath samples were collected before and immediately after breathing oxygen. The research was approved by the institutional review board of Saint Vincents Catholic Medical Centers of New York and all subjects gave their signed informed consent to participate. 


\section{Breath volatile organic compounds collection and assay}

The method has been described previously [14]. A portable breath collection apparatus was employed to capture the volatile organic compounds (VOCs) in $1.0 \mathrm{~L}$ breath and in $1.0 \mathrm{~L}$ room air on to separate sorbent traps. Subjects wore a noseclip while breathing in and out of the disposable mouthpiece of the apparatus for $2.0 \mathrm{~min}$. VOCs in the sorbent traps were analysed by automated thermal desorption, gas chromatography and mass spectroscopy.

\section{Derivation of breath methylated alkane contour}

Three-dimensional surface plots of C4 C20 n-alkanes and their monomethylates were obtained using a previously described method [13]. In summary, data were obtained from VOC peaks observed on breath chromatograms: $\mathrm{Vb}=$ area under the curve (AUC) of alkane or methylated alkane; $\mathrm{Ib}=\mathrm{AUC}$ of internal standard used to calibrate the instrument $(0.25 \mathrm{~mL}, 2$ parts per million 1-bromo-4-fluoro-benzene (Supelco, Bellefonte, PA, USA)). Va and Ia were corresponding AUCs derived from chromatograms of room air. The alveolar gradient $[15,16]$ of each VOC was then determined as follows:

$$
\text { alveolar gradient }=\mathrm{Vb} / \mathrm{Ib}-\mathrm{V}_{\mathrm{a}} / \mathrm{Ia}
$$

Mean alveolar gradients of these VOCs were computed for all subjects before and after breathing oxygen and displayed in surface plots showing the carbon chain length on the x-axis, the methylation site on the z-axis, and the mean alveolar gradient on the y-axis.

\section{Analysis of data}

Paired t-tests were employed to compare the preand post-oxygenation alveolar gradients of C4-C20 n-alkanes and their monomethylates, as well as the volume under the curve of the BMACs.

\section{Results}

All subjects completed the study without adverse effects. The mean pre- and post-oxygenation BMACs are shown in figure 1. There was a global increase in the alveolar gradients of $\mathrm{C} 4-\mathrm{C} 20 \mathrm{n}$-alkanes and their monomethylated derivatives, as shown by a significant increase in the mean volume under the curve of the BMAC (fig. 2). The alveolar gradients of three BMAC VOCs, 3-methyltridecane, 3-methylundecane and 5 -methylnonane, were significantly $(\mathrm{p}<0.05)$ increased after oxygenation.

\section{Discussion}

Normal healthy volunteers significantly increased their output of a broad spectrum of breath markers of oxidative stress after breathing $28 \%$ oxygen for $30 \mathrm{~min}$. These findings are noteworthy, first, because previous studies of hyperoxia have yielded contradictory results, and secondly, because of the clinical implications of monitoring oxygen therapy.

The BMAC comprises a set of 107 different $\mathrm{C} 4-\mathrm{C} 20$ alkanes and monomethylated alkanes, each of which is an independent marker of oxidative stress. These markers were evaluated before and after oxygen breathing in two different ways: first, by comparing changes in the abundance of individual VOCs, and second, by comparing their integrated total abundance as the mean volume under the curve of the BMAC. Three of these individual markers were significantly increased by oxygen breathing, and there was also a significant increase in their integrated total abundance, the mean volume under the curve of the BMAC.

Many different markers of oxidative stress have been proposed, including malonaldehyde and conjugated dienes in the blood, as well as alkanes and hydrogen peroxide in the breath [3,4]. Despite their chemical diversity, all of these markers of oxidative stress share one common feature: all are downstream metabolites of parent molecules that have been oxidised by ROS. However, no comprehensive study has yet compared the sensitivity and specificity of these different markers or estimated their relative value as clinical research tools. Consequently, it would be desirable to validate the current findings in future studies by comparing the effects of oxygen breathing on other markers of oxidative stress.

Previous studies of the effects of hyperoxia on other markers of oxidative stress have yielded inconsistent findings. Hyperoxia in normal humans breathing $100 \%$ oxygen has been reported to increase breath levels of pentane as well as serum malondialdehyde $[9,10]$ but induced hyperoxia in newborn lambs elicited no significant changes in either breath ethane or pentane [17]. In preterm infants ventilated for respiratory distress syndrome, DRURY et al. [18] found no correlation between fractional inspired oxygen concentration and exhaled pentane, but NYCYK et al. [19] found an association between high-peak pentane exhalation and low gestational age, mortality, intraventricular haemorrhage and retinopathy of prematurity.

The inconsistency of these findings has led to controversy as to whether or not oxygen breathing is the real cause of increased oxidative stress. However, these inconsistencies may have resulted, at least in part, from technically inadequate measurements. While breath testing may appear intuitive and simple, its apparent simplicity conceals a number of formidable obstacles that can result in technical failure. Technical failures in breath testing for markers of oxidative stress are of three main types. 1) Inadequate breath collection technique and failure to correct for VOCs present in background air. CAILLEUX and Allain [20] observed that pentane is a normal constituent of room air. Hence, any breath test that is not corrected for VOCs inspired from room air will generate erroneous results. 2) Inadequate analytic technique. Kohlmuller and Kochen [21] found that commonly used chromatography columns failed to separate n-pentane and isoprene, and they concluded that reports of breath pentane in the literature had 

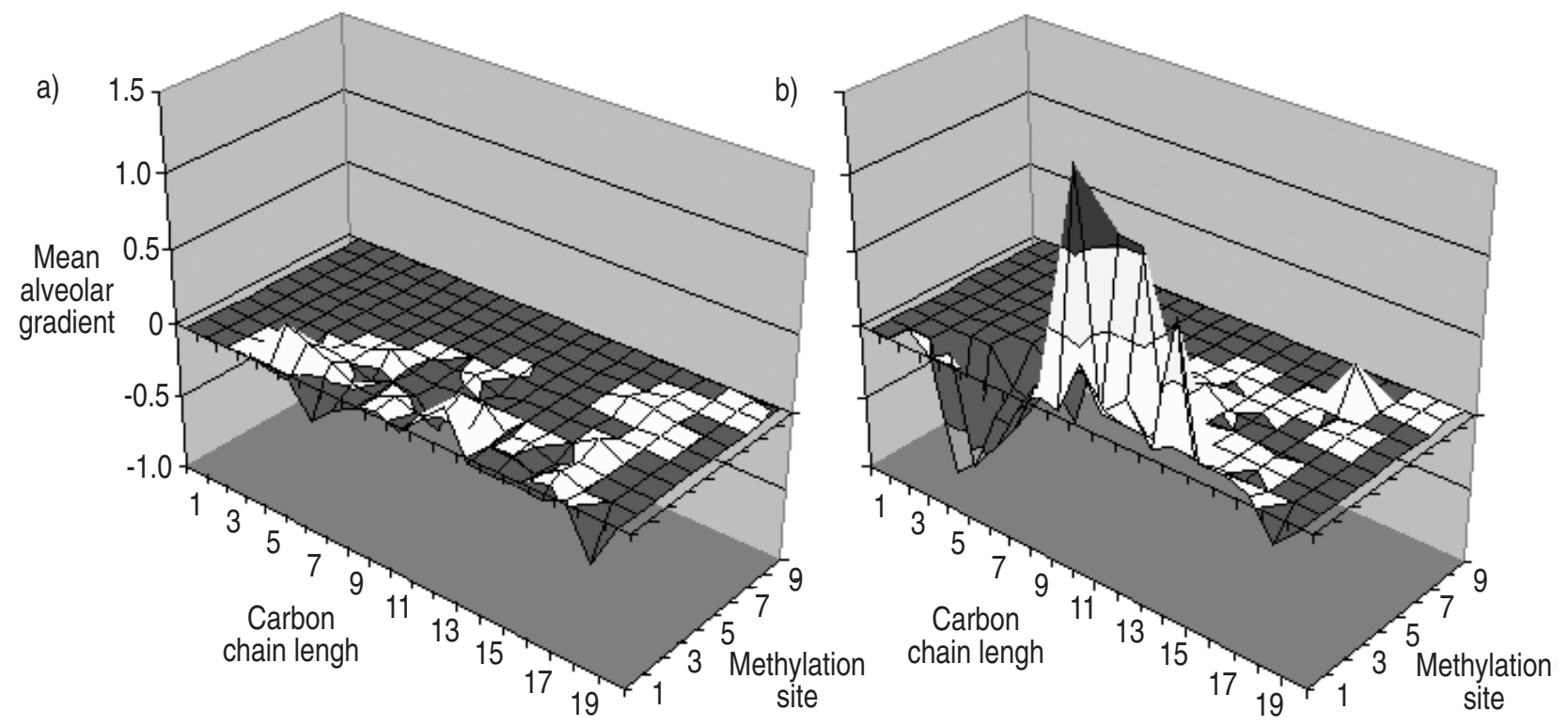

Fig. 1. - Breath methylated alkane contours (BMACs) before and after oxygenation. The BMAC is a three-dimensional surface plot of the results of the breath test for volatile organic compounds (VOC). The mean alveolar gradient (concentration in breath-concentration in room air) is shown on the vertical axis for $\mathrm{C} 4-\mathrm{C} 20$ alkanes and their monomethylated derivatives. The horizontal axes identify the specific VOC (e.g. the combination of carbon chain length $=4$ and methylation site $=2$ corresponds to 2 -methylbutane). It is apparent that the mean alveolar gradients of several VOCs were increased by oxygenation. There was a significant global increase in volume under the curve (see fig. 2).

actually measured isoprene or a mixture of pentane and isoprene. 3) Failure to recognise the inherent limitations of employing a single-breath marker of oxidative stress. Ethane and pentane in breath may be measured (or mismeasured) quickly and easily. This has resulted in a widespread misconception that ethane and pentane are the sole markers of oxidative stress in breath, while the reality is that they are only two members of a much larger constellation of markers.

These potential pitfalls were avoided through use of the breath test in this study. First, subambient temperature programming was employed in the gas

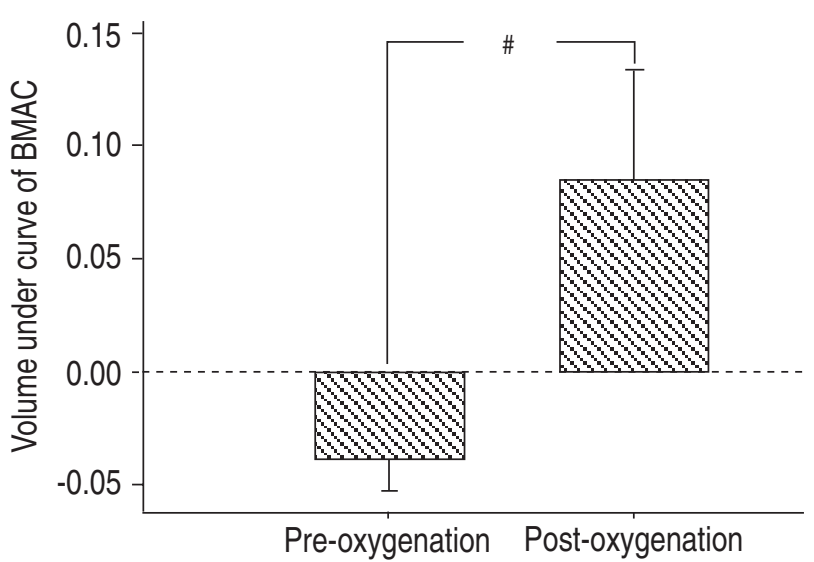

Fig. 2.-Mean volume under the curve (VUC) of the breath methylated alkane contour (BMAC) before and after oxygenation. This diagram displays the mean VUC of the BMACs in figure 1 (error bar=SEM). The change demonstrates a significant global increase in the volatile markers of oxidative stress that comprise the BMAC. chromatograph. This provided superior separation of VOCs which might otherwise have co-eluted with one another, and the purity of every VOC in the BMAC in all subjects was confirmed by mass spectroscopy $[14,15]$. Secondly, for every subject studied, two samples (one of breath and one of room air) were routinely collected and assayed. This made it possible to determine the alveolar gradient of a VOC (abundance in breath-abundance in room air), which varies with the difference between the rates of synthesis and clearance of a VOC in the body [14, 15]. Thirdly, the BMAC was employed as a set of markers of oxidative stress. The advantage of the BMAC is that it employs the range of $\mathrm{C} 4-\mathrm{C} 20$ alkanes and their monomethylated derivatives as a composite set of markers of oxidative stress. In this set, pentane is only one VOC in a spectrum of 107 different VOCs, and the BMAC is less likely to be skewed by the relative increase or decrease of a single VOC.

Oxidative stress caused by oxygen toxicity may have adverse effects on virtually every organ of the body. This has been most extensively demonstrated in neonates, where oxygen toxicity has long been linked to retrolental fibroplasia and bronchopulmonary dysplasia [22]. In adults, prolonged exposure to hyperbaric oxygen can cause central nervous system toxicity, atelectasis, pulmonary oedema and seizures [23]. The adverse effects of normobaric hyperoxia are less well defined, probably because of the time lag between injuries sustained at the molecular level and subsequent pathological changes. However, increased oxidative stress has been shown to modify the structure of proteins [24] and DNA [25] and has been linked to an increased risk of cancer [26, 27] and chronic degenerative diseases, including atherosclerosis, heart 
failure, Parkinson's disease and Alzheimer's disease [27-30].

The authors conclude that breathing $28 \%$ oxygen for $30 \mathrm{~min}$ caused a significant increase in oxidative stress in normal volunteers. The clinical significance of this finding is not known, since a transient increase in oxidative stress may not necessarily progress to detectable cellular dysfunction or damage. However, in view of the well-documented adverse effects of prolonged oxidative stress, these findings reinforce what clinicians already know well: the routine use of oxygen to induce hyperoxia is potentially hazardous and it should be reserved only for situations in which it has demonstrated clinical benefit.

\section{References}

1. Knight JA. Free radicals: their history and current status in aging and disease. Ann Clin Lab Sci 1998; 28: 331-346.

2. Bostek CC. Oxygen toxicity: an introduction. AANA J 1989; 57: 231-237.

3. Kneepkens CM, Ferreira C, Lepage G, Roy CC. The hydrocarbon breath test in the study of lipid peroxidation: principles and practice. Clin Invest Med 1992; 15: 163-186.

4. Kneepkens CM, Lepage G, Roy CC. The potential of the hydrocarbon breath test as a measure of lipid peroxidation. Free Radic Biol Med 1994; 17: 127-160.

5. Ambrosone CB. Oxidants and antioxidants in breast cancer. Antioxid Redox Signal 2000; 2: 903-917.

6. Onorato JM, Thorpe SR, Baynes JW. Immunohistochemical and ELISA assays for biomarkers of oxidative stress in aging and disease. Ann $N$ Y Acad Sci 1998; 854: 277-290.

7. Chandra M, Chandra N, Agrawal R, Kumar A, Ghatak A, Pandey VC. The free radical system in ischemic heart disease. Int J Cardiol 1994; 43: 121-125.

8. Emelyanov A, Fedoseev G, Abulimity A, et al. Elevated concentrations of exhaled hydrogen peroxide in asthmatic patients. Chest 2001; 120: 1136-1139.

9. Morita S, Snider MT, Inada Y. Increased N-pentane excretion in humans: a consequence of pulmonary oxygen exposure. Anesthesiology 1986; 64: 730-733.

10. Loiseaux-Meunier MN, Bedu M, Gentou C, Pepin D, Coudert J, Caillaud D. Oxygen toxicity: simultaneous measure of pentane and malondialdehyde in humans exposed to hyperoxia. Biomed Pharmacother 2001; 55: 163-169.

11. Roberts RJ, Rendak I, Bucher JR. Lipid peroxidation in the newborn rat: influence of fasting and hyperoxia on ethane and pentane in expired air. Dev Pharmacol Ther 1983; 6: 170-178.

12. Turanlahti M, Pesonen E, Lassus P, Andersson S. Nitric oxide and hyperoxia in oxidative lung injury. Acta Paediatr 2000; 89: 966-970.
13. Phillips M, Cataneo RN, Greenberg J, Gunawardena R, Naidu A, Rahbari-Oskoui F. Effect of age on the breath methylated alkane contour, a display of apparent new markers of oxidative stress. $J$ Lab Clin Med 2000; 136: 243-249.

14. Phillips M. Method for the collection and assay of volatile organic compounds in breath. Anal Biochem 1997; 247: 272-278.

15. Phillips M, Herrera J, Krishnan S, Zain M, Greenberg $\mathrm{J}$, Cataneo RN. Variation in volatile organic compounds in the breath of normal humans. J Chromatogr B Biomed Sci Appl 1999; 729: 75-88.

16. Phillips M, Greenberg J, Sabas M. Alveolar gradient of pentane in normal human breath. Free Radic Res 1994; 20: 333-337.

17. Hansen TN, Smith CV, Gest AL, Smith HW, Giesler M. Biochemical manifestations of oxygen toxicity in the newborn lamb. Pediatr Res 1990; 28: 613-617.

18. Drury JA, Nycyk JA, Cooke RW. Pentane measurement in ventilated infants using a commercially available system. Free Radic Biol Med 1997; 22: 895-900.

19. Nycyk JA, Drury JA, Cooke RW. Breath pentane as a marker for lipid peroxidation and adverse outcome in preterm infants. Arch Dis Child Fetal Neonatal Ed 1998; 79: F67-F69.

20. Cailleux A, Allain P. Is pentane a normal constituent of human breath? Free Radic Res Commun 1993; 18: 323-327.

21. Kohlmuller D, Kochen W. Is n-pentane really an index of lipid peroxidation in humans and animals? A methodological reevaluation. Anal Biochem 1993; 210: 268-276.

22. Saugstad OD. Chronic lung disease: oxygen dogma revisited. Acta Paediatr 2001; 90: 113-115.

23. Jenkinson SG. Oxygen toxicity. New Horiz 1993; 1: 504-511.

24. Naskalski JW, Bartosz G. Oxidative modifications of protein structures. Adv Clin Chem 2000; 35: 161-253.

25. De Flora S, Izzotti A, Randerath $\mathrm{K}$, et al. DNA adducts and chronic degenerative disease. Pathogenetic relevance and implications in preventive medicine. Mutat Res 1996; 366: 197-238.

26. Dreosti IE. Nutrition, cancer, and aging. Ann $N Y$ Acad Sci 1998; 854: 371-377.

27. Loft S, Poulsen HE. Cancer risk and oxidative DNA damage in man. J Mol Med 1996; 74: 297-312.

28. Hemnani T, Parihar MS. Reactive oxygen species and oxidative DNA damage. Indian J Physiol Pharmacol 1998; 42: 440-452.

29. Gotz ME, Kunig G, Riederer P, Youdim MB. Oxidative stress: free radical production in neural degeneration. Pharmacol Ther 1994; 63: 37-122.

30. Perry G, Smith MA. Is the lesion produced by oxidation a central part in the pathogenesis of Alzheimer's disease? Neurologia 1999; 14: 78-84. 